\title{
Eotaxin-3 and a uniquely conserved gene- expression profile in eosinophilic esophagitis
}

\author{
Carine Blanchard, ${ }^{1}$ Ning Wang, ${ }^{1,2}$ Keith F. Stringer, ${ }^{3}$ Anil Mishra, ${ }^{1}$ Patricia C. Fulkerson, ${ }^{4}$
} J. Pablo Abonia, ${ }^{1}$ Sean C. Jameson, ${ }^{1}$ Cassie Kirby, ${ }^{1}$ Michael R. Konikoff, ${ }^{5}$ Margaret H. Collins, ${ }^{3}$ Mitchell B. Cohen, ${ }^{5}$ Rachel Akers, ${ }^{6}$ Simon P. Hogan, ${ }^{1}$ Amal H. Assa'ad, ${ }^{1}$ Philip E. Putnam, ${ }^{5}$ Bruce J. Aronow, ${ }^{7}$ and Marc E. Rothenberg ${ }^{1}$

\begin{abstract}
1Division of Allergy and Immunology, ${ }^{2}$ Division of Human Genetics, and ${ }^{3}$ Division of Pathology and Laboratory Medicine, Cincinnati Children's Hospital Medical Center, Department of Pediatrics, University of Cincinnati College of Medicine, Cincinnati, Ohio, USA. ${ }^{4}$ Department of Molecular Genetics and Biochemistry, University of Cincinnati College of Medicine, Cincinnati, Ohio, USA. ${ }^{5}$ Division of Gastroenterology, Hepatology and Nutrition, ${ }^{6}$ Center for Epidemiology and Biostatistics, and 7Division of Pediatric Informatics, Cincinnati Children's Hospital Medical Center Department of Pediatrics, University of Cincinnati College of Medicine, Cincinnati, Ohio, USA.
\end{abstract}

\begin{abstract}
Eosinophilic esophagitis (EE) is an emerging disorder with a poorly understood pathogenesis. In order to define disease mechanisms, we took an empirical approach analyzing esophageal tissue by a genome-wide microarray expression analysis. EE patients had a striking transcript signature involving $1 \%$ of the human genome that was remarkably conserved across sex, age, and allergic status and was distinct from that associated with non-EE chronic esophagitis. Notably, the gene encoding the eosinophil-specific chemoattractant eotaxin-3 (also known as CCL26) was the most highly induced gene in EE patients compared with its expression level in healthy individuals. Esophageal eotaxin-3 mRNA and protein levels strongly correlated with tissue eosinophilia and mastocytosis. Furthermore, a single-nucleotide polymorphism in the human eotaxin-3 gene was associated with disease susceptibility. Finally, mice deficient in the eotaxin receptor (also known as CCR3) were protected from experimental EE. These results implicate eotaxin-3 as a critical effector molecule for EE and provide insight into disease pathogenesis.
\end{abstract}

\section{Introduction}

Eosinophilic esophagitis (EE) is an emerging worldwide disease, as documented by recent case series from Switzerland, Australia, Canada, Japan, England, and the US (1-4). Of concern, EE appears to be a growing health problem with an annual incidence of at least 1 in 10,000 children (5). The primary symptoms of EE (chest and abdominal pain, dysphagia, heartburn, vomiting, and food impaction) are also observed in patients with chronic esophagitis (CE) including gastroesophageal reflux disease (GERD) (6-8). However, in contrast to GERD, EE occurs more frequently in males $(80 \%)$, appears to have a common familial form, has a high rate of associated atopic disease $(70 \%)$, and is typically associated with a normal $\mathrm{pH}$ probe recording of the esophagus $(5,9,10)$. Distinguishing EE from GERD is important since EE patients do not respond to anti-GERD therapy but may respond to anti-inflammatory therapy and/or allergen elimination $(6,11-13)$. Whereas both GERD and EE are associated with esophageal eosinophils, the level of eosinophils in $\mathrm{EE}$ is much higher, greater than 24 eosinophils per high-power field (hpf) $(\times 400)(11)$; the normal esophagus is devoid of eosinophils $(14,15)$. However, whether GERD and EE represent a continuum, with EE being a more severe manifestation, has not been adequately addressed. A clearer differentiation between these various esophagitis states is clearly needed.

Dissection of experimental EE models in mice has revealed that EE can be triggered by both food and aeroallergens (16,

Nonstandard abbreviations used: CCR, CC chemokine receptor; CE, chronic esophagitis; CLC, Charcot-Leyden crystal; EE, eosinophilic esophagitis; FDR, false discovery rate; GERD, gastroesophageal reflux disease; hpf, high-power field; MBP, major basic protein; NL, normal; SNP, single-nucleotide polymorphism.

Conflict of interest: The authors have declared that no conflict of interest exists. Citation for this article: J. Clin. Invest. 116:536-547 (2006). doi:10.1172/JCI26679.
17). However, nearly $25 \%$ of people with EE are nonatopic individuals with no identifiable allergic sensitization $(2,5,9,18)$. It is critically important to understand the relationship between the allergic and nonallergic variants of EE; whether allergic and nonallergic esophagitis involves similar effector pathways has significant implications for therapeutic strategies. Murine modeling has established that EE is a Th2-associated disease $(17,19)$. IL-5 is required for disease pathogenesis in an experimental model (16); indeed, humanized anti-IL-5 appears to be effective in an early clinical study (20). Human EE is associated with overproduction of the Th2 cytokines IL-4 and IL-13 $(18,21)$. Although these Th2 cytokines have been implicated, the mechanism by which they lead to esophageal eosinophilia is unclear. While IL-4 and IL-13 are known to induce the eosinophil-specific eotaxin chemokines (e.g., eotaxin-1, eotaxin-2, and eotaxin-3) (22-25), their role has remained elusive, since they have not yet been demonstrated to be overproduced in EE, and eotaxin-1-deficient mice only develop a modest attenuation of experimental EE $(16,19)$.

In an effort to provide unbiased insight into disease pathogenesis, we took an empirical approach involving expression profiling of esophageal biopsy tissue from patients with $\mathrm{EE}$ and comparison of this tissue with tissue from patients with CE, as well as healthy controls (referred to herein as NL [normal]). Whole-genome-wide expression analysis uncovered a striking EE transcript signature that was similar across patients' sex and age, but distinct from CE. Notably, allergic and nonallergic variants of EE were found to have a conserved esophageal transcriptome indicating overlapping effector pathways in the diseased tissue. Furthermore, the most highly induced transcript in EE was eotaxin-3; notably, levels of eotaxin-3 strongly correlated with disease severity, and a singlenucleotide polymorphism (SNP) in the eotaxin-3 gene was associated with disease susceptibility. Lastly, the importance of this 
A

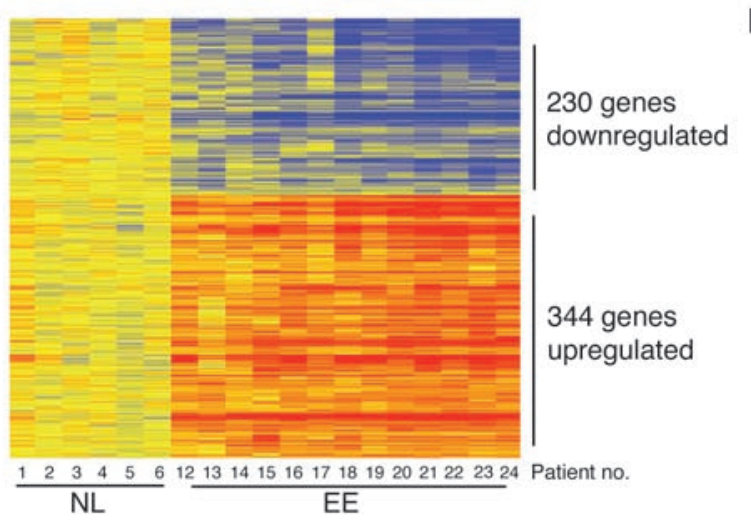

B

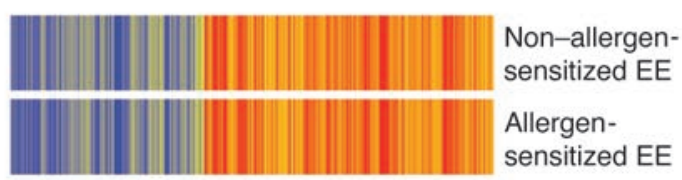

C

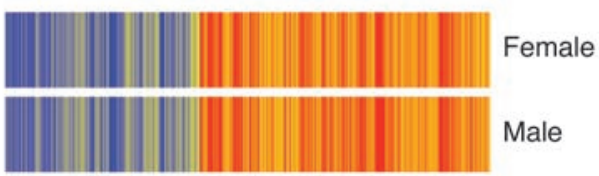

D

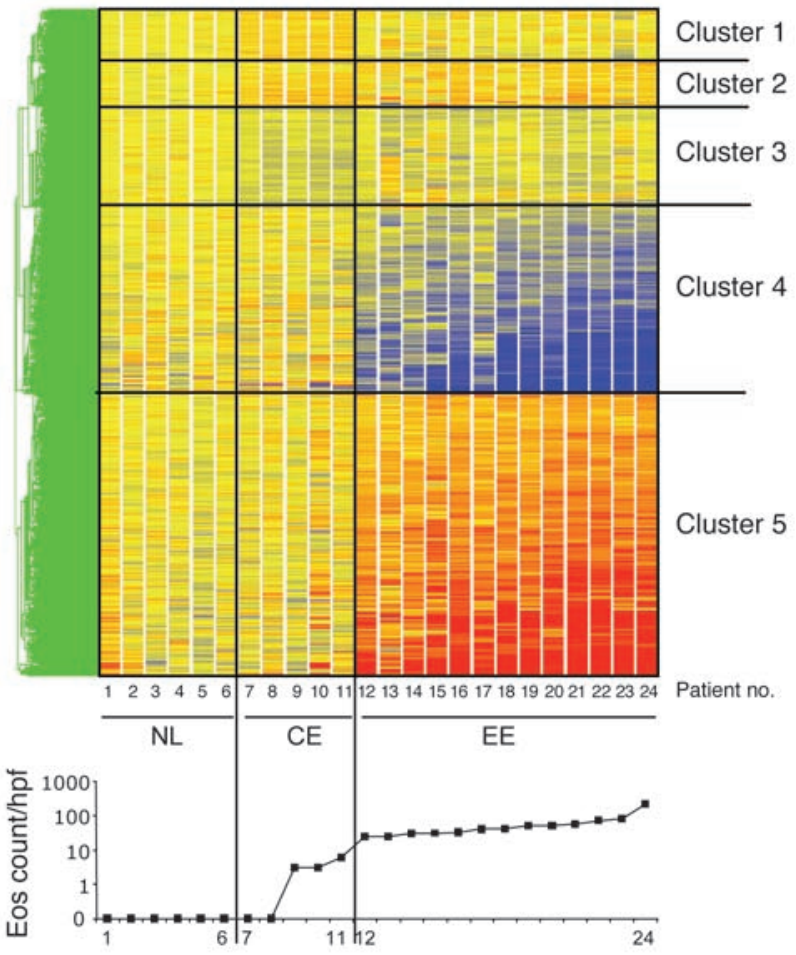

\section{Figure 1}

Microarray analysis of the transcripts expressed in esophageal biopsies. RNA from each patient was subjected to chip analysis using Affymetrix Human Genome U133 Plus 2.0 GeneChips. The normal (NL) group is composed of 6 individuals (numbers 1-6), the CE group is represented by 5 patients (numbers 7-11), and 13 patients (numbers 12-24) are in the EE group, as reported in Supplemental Table 1. (A) The 574 genes differentially expressed $(P<0.01)$ in the EE group compared with normal healthy patients have been ordered (standard correlation); upregulated genes are represented in red and downregulated genes in blue. The magnitude of the gene changes is proportional to the darkness of the color. Each column represents a separate individual and each line a gene. (B and C) EE transcript signature is presented as a function of the allergic status and sex of EE patients. Average expression of the transcripts of the EE signature is depicted in the non-allergen-sensitized $(n=4)$ and allergen-sensitized EE patients $(n=9)(B)$ and in female $(n=5)$ and male EE patients $(n=8)$ (C). (D) The 574 genes expressed significantly differently $(P<0.01)$ in the EE group compared with normal healthy patients and the 228 genes expressed differently $(P<0.01)$ in the CE group compared with normal healthy patients have been analyzed by cluster analysis and ordered (distance) using GeneSpring software. Clusters 1 , 2, and 3 highlight the CE transcripts, and clusters 4 and 5 highlight the EE transcripts. The eosinophil (Eos) count in each patient is shown in the lower panel.

pathway was demonstrated by the protection from experimental EE observed in mice harboring a genetic deletion in the eotaxin receptor (CC chemokine receptor 3 [CCR3]).

\section{Results}

EE transcript signature. Esophageal biopsy samples derived from individual patients (see Supplemental Table 1; supplemental material available online with this article; doi:10.1172/JCI26679DS1) were subjected to whole-genome-wide transcript expression profile analysis using oligonucleotide-based DNA microarray chips. Of the 54,681 transcripts represented on these microarrays, 574 transcripts (Supplemental Table 2) were differently expressed $(P<0.01)$ in the EE patients versus normal (NL) biopsy samples; thus, approximately $1 \%$ of the whole human genome transcripts define the transcript signature of EE. Hierarchical clustering of the signal intensities of the individual transcripts in each group showed a high similarity of transcript expression patterns among EE patients (Figure 1A). Of these, 344 transcripts were expressed more abundantly and 230 were expressed less abundantly in the EE patients compared with the NL group (Figure 1A). Gene ontology analysis of the EE transcript signature (Supplemental Table 3) revealed that the overexpressed genes were frequently involved in cell communication (26\%), signal transduction (22\%), response to external stimulus (20\%), immune response (16\%), and response to stress $(11 \%)$. In contrast, the downregulated genes were composed of a distinct family of functional groups (Supplemental Table 4). The complete annotation of all the transcripts expressed differently in EE patients compared with the NL group is presented in Supplemental Table 5.

While there are numerous related families of dysregulated genes, it is notable that 5 mast cell genes were highly induced (including carboxypeptidase A3, 13-fold; high-affinity IgE receptor [FcERI], 4-fold; and mast cell tryptase- $\alpha, 6$-fold). Interestingly, the maximum mast cell count per hpf was significantly increased $(13.8 \pm 1.8$ cells per hpf, mean $\pm \mathrm{SD}, n=13, P<0.0005)$ in biopsies from EE patients compared with NL $(4.6 \pm 0.3, n=6)$ and CE patients $(5.8 \pm 1.1$, $n=5$ ), as shown in Supplemental Table 1 and Figure 2A. Whereas evidence of extracellular eosinophil granule constituents (indicative of eosinophil activation) was appreciated in most EE tissue sections, there was no dramatic evidence of mast cell degranulation based on the absence of extracellular mast cell constituents (data not shown). Further assessment revealed that mast cells and 
A
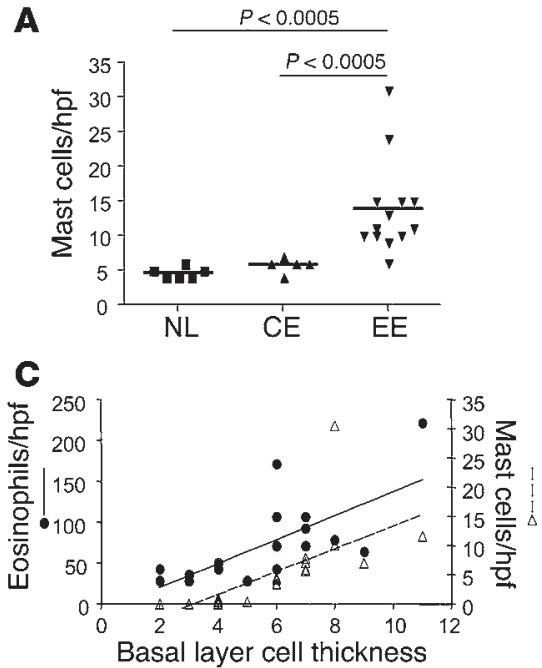

B

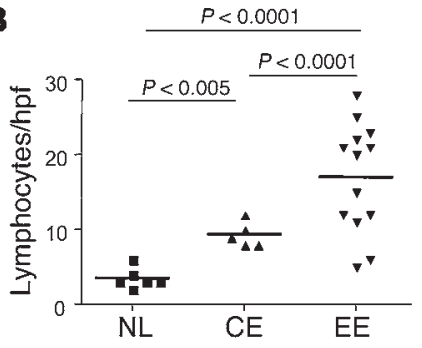

D

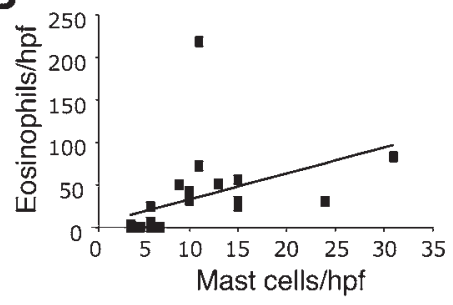

\section{Figure 2}

Mast cell and lymphocyte counts in NL, CE, and EE patients. (A) The maximum mast cell count per hpf was assessed in patients 1-24 using immunohistochemistry. Biopsies were stained using monoclonal anti-human tryptase. (B) The maximum lymphocyte count per hpf was assessed in patients 1-24 on H\&E staining. $P$ values were calculated using the Welch $T$ test (A and B). (C) The correlations between basal layer cell thickness and both maximum eosinophil level $\left(r^{2}=0.47\right.$, $P<0.0005)$ and mast cell level $\left(r^{2}=0.51, P<0.0001\right)$ are shown. (D) The maximum eosinophil levels are presented as a function of maximum mast cell levels $\left(r^{2}=0.18, P<0.05\right)$. $P$ values were based on Pearson correlation (C and $\mathbf{D})$. eosinophils correlated with basal layer hyperplasia $(P<0.0001$ and $P<0.0005$, respectively) (Figure 2C). Mast cells also correlated with eosinophil counts $(P<0.05)$ (Figure $2 \mathrm{D})$. Arachidonic acid metabolism genes and regulators of cell growth and maintenance were also strikingly represented, as shown in Supplemental Table 5.

The identification of an EE transcript signature provided a valuable opportunity to uncover critical aspects of disease pathogenesis. First, we were interested in determining whether the allergic and nonallergic variants of EE had different transcript profiles. When we compared the full EE transcript profile between allergen-sensitized and non-allergen-sensitized EE patients, there was nearly complete overlap in the transcripts (Figure 1B) of the EE signature genome defined in Supplemental Table 5. Only 10 genes were differently expressed, and only 1 was changed at least 2 -fold (lymphocyte antigen 75 [LY75]; 2-fold increase in allergic). The human LY75 molecule has previously been shown to affect IL-4 signaling (26). When EE patients were divided into allergic and nonallergic groups based on aeroallergen or food allergen sensitization or history of allergic disease, no significant differences (false discovery rate $[\mathrm{FDR}]<0.05)$ were observed between the positive and negative groups. As shown in Supplemental Table 1, a dramatic overlap exists between patients sensitive to food and aeroallergens and patients with a history of allergic disease (food anaphylaxis, allergic rhinitis, asthma, or atopic dermatitis). Second, we aimed to determine whether EE patients had variable expression of genes dependent on their age. Of the 574 dysregulated transcripts, no gene correlated with patient age within the EE transcript profile. As a control, outside of the EE transcript signature, there were 334 genes that correlated to patient age (Pearson correlation test with $P<0.01)$. As such, pediatric EE and adolescent EE do not have dramatic differences in the transcript signature, suggesting that the pathogenesis might be largely conserved across age differences. Finally, since EE is more common in males, we were interested in determining whether the EE transcript profile was different between males and females. Notably, only 1 gene of the EE transcript signature (tyrosine kinase receptor $B$ ) was dependent on the patients' sex (3-fold increase in females) (Figure 1C). Outside of the EE transcript signature, there were 434 genes that differed between male and female EE (Supplemental Table 2; $P<0.005$ ). Interestingly, some patients were on preexisting therapy (includ- ing proton pump inhibitors and leukotriene receptor antagonists) at the time of endoscopy, as indicated in Supplemental Table 1. However, no significant differences in the identified transcripts were found (FDR < 0.05), as shown in Supplemental Table 2. Taken together, these results demonstrate that the EE transcript profile is remarkably conserved between patients despite differences in sex, age, and allergic status.

Differentiation of EE and CE. We compared the transcript expression profile in patients who presented with symptoms of EE but were found to have CE. Cluster analysis was performed to stratify dynamic genes into related subgroups (Figure 1D). In Figure 1D, the EE transcripts are seen in clusters 4 and 5; the CE transcripts are seen in clusters 1,2, and 3. CE patient biopsies showed an expression profile close to that of the NL patient biopsies. The CE transcript esophageal samples contained only 228 dynamic transcripts (Supplemental Table 2), approximately $0.4 \%$ of the tested genome, compared with NL samples $(P<0.01)$. Indeed, there were significant changes in tissue pathology including lymphocyte levels and the degree of epithelial cell hyperplasia in CE versus NL samples (Supplemental Table 1 and Figure 2B). These 228 transcripts (114 overexpressed, shown in combined clusters 1 and 2, and 114 downregulated in cluster 3 ) were rich in genes involved in intracellular cascades (10\%) and biosynthesis (10\%) (both in cluster 1 ) and cell growth and maintenance (23\%) (in cluster 2) (Supplemental Table 6). Notably, no transcript was modified 5-fold or more in CE compared with NL samples (Figure 3). In contrast, 124 genes were modified 5-fold or more in EE compared with NL samples, including 42 transcripts that were modified 10 -fold or more in EE; these dysregulated genes are shown in Figure 3. To define genes that could distinguish EE from CE, we directly compared the EE and the CE transcriptomes. There was an overlap of only 40 genes between EE and CE (mainly in cluster 2) (Figure 1D and Supplemental Table 7), and only 5 of these overlapped with the EE transcript signature. Taken together, these results suggest that $\mathrm{EE}$ and CE are unlikely to share the same disease process. Furthermore, the identification of strongly induced genes that distinguish EE from CE (Figure 3) defines potential diagnostic criteria that are likely to distinguish these forms of esophagitis.

Disease severity index. We were interested in analyzing the EE transcriptome as a function of disease severity. We hypothesized that 


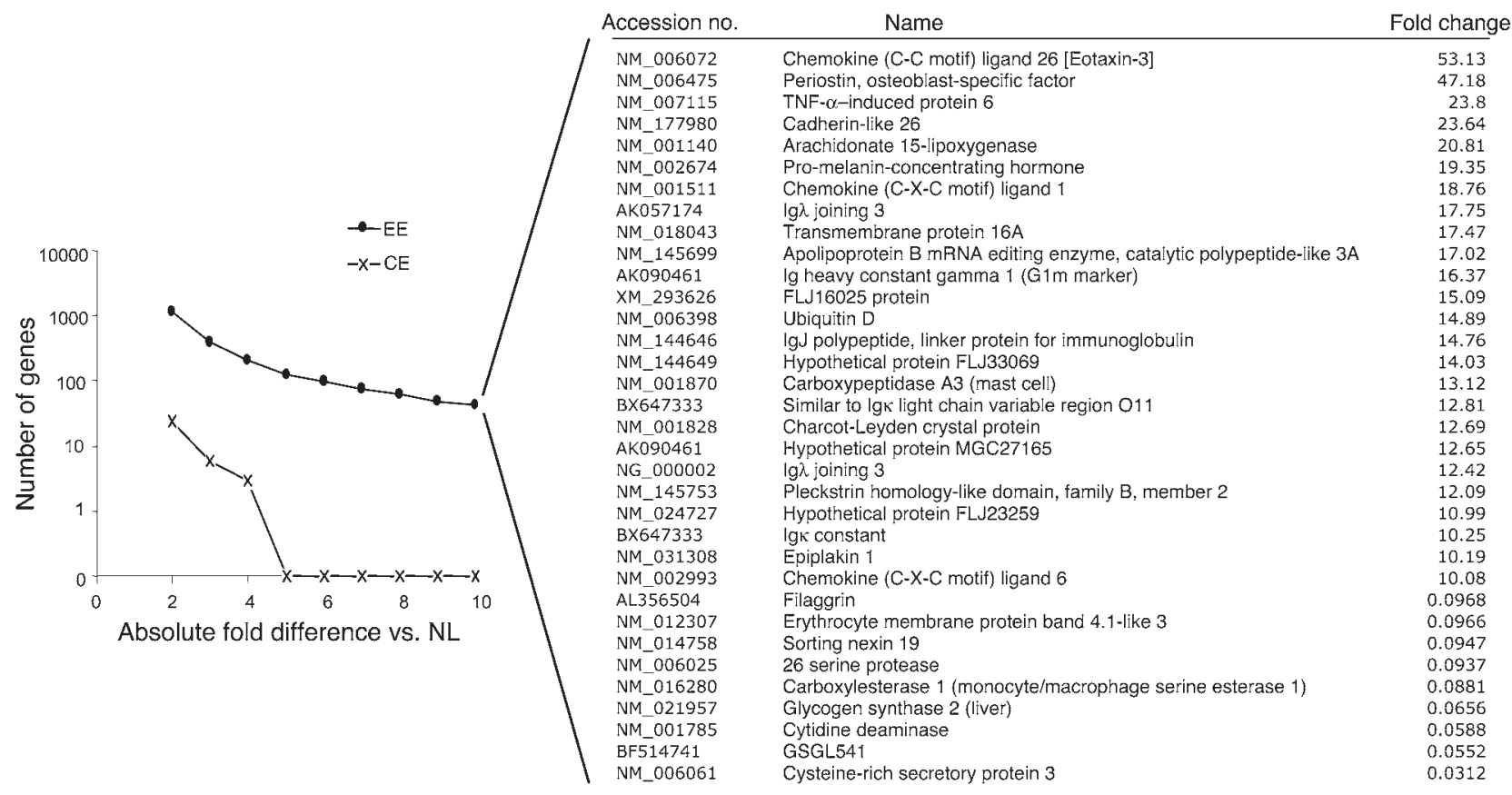

Figure 3

Numbers of modified genes and their fold change in EE and CE. The average gene-expression levels in the EE and CE groups have been compared with that in the NL group. The number of genes that changed at least 10-fold is shown. The list of the 42 transcripts that were modified at least 10-fold in the EE compared with the NL group and their GenBank accession numbers is shown. The list includes 8 transcripts that were found twice in the EE transcriptome.

the number and magnitude of modified genes might be directly related to histological severity. We thus aimed to determine whether eosinophil levels would correlate with the number of altered genes. The population of patients with EE had peak eosinophil levels that varied between 24 and 218 eosinophils per hpf (Figure 1D). The number of dysregulated genes increased between eosinophil levels of 0 and 83 (Figure 4A). Similarly, the magnitude of gene changes directly correlated with eosinophil levels (Figure 1D). As such, 321 genes $(55 \%)$ of the EE transcript signature are among the 1,943 genes that most correlated $(P<0.005)$ with eosinophil levels (Figure $4 \mathrm{~B})$. Interestingly, the mast cell gene signature was located in the 321 genes that correlated with eosinophil numbers.

Eotaxin-3 expression in the esophagus. Within the EE transcript signature, the gene with the greatest change was eotaxin-3, which was induced 53-fold (Figure 3). Other relevant eosinophil chemokines, such as eotaxin-1 and eotaxin-2, were induced less than 2-fold in EE samples. Given that our analysis was a whole-genome-wide approach and that the gene with the largest expression change was a relevant, specific, and potentially important eosinophil chemoattractant (24), we prioritized investigation of this find-

\section{Figure 4}

Correlation between eosinophil count and number of genes modified. (A) The number of genes expressed differently is presented as a function of the eosinophil count. The number of genes that changed at least 10 -fold is plotted as a function of the maximum number of eosinophils in the biopsies (patients 1-23). A trendline (black line) has been inserted $\left(r^{2}=0.73, P<0.05\right)$. (B) The overlap between the EE transcript signature (574 genes) and the 1,943 genes that most correlated $(P \leq 0.005)$ with the number of eosinophils is presented in a Venn diagram. ing. Using real-time PCR analysis (LightCycler), a mean 53-fold increase in eotaxin-3 mRNA compared with that in NL samples was observed (Figure 5A). Modest changes in eotaxin-1 (Figure 5B) and eotaxin-2 (Figure 5C) were observed in EE patients, although there was some variability among patients.

It was next important to determine whether the level of eotaxin-3 correlated with eosinophil levels in esophageal samples. As shown in Figure 6A, 2 methods (LightCycler quantification and microarray analysis) revealed a strong correlation between eotaxin-3 mRNA and peak eosinophil counts $(P<0.005)$. In addition, eotaxin-3 mRNA correlated with mast cell levels based on microarray analysis $(P<0.005)$ and on LightCycler quantification $(P<0.05)$ (Figure 6B).

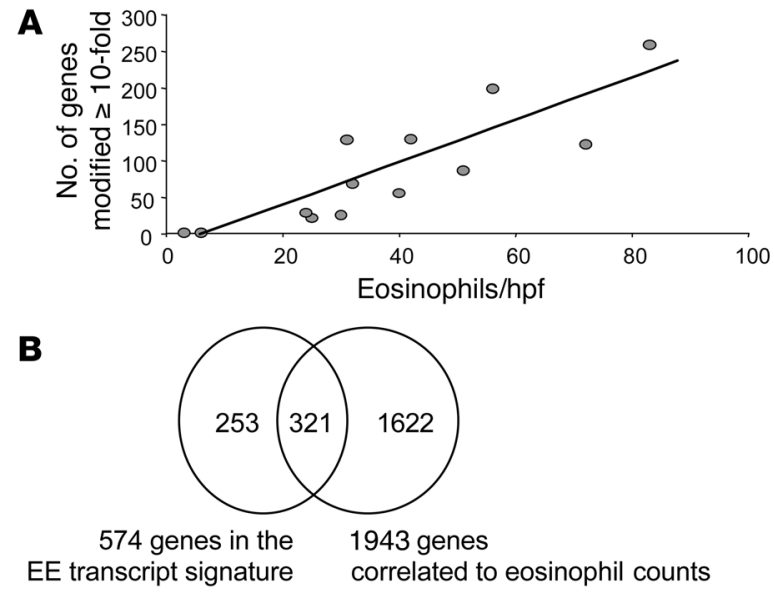



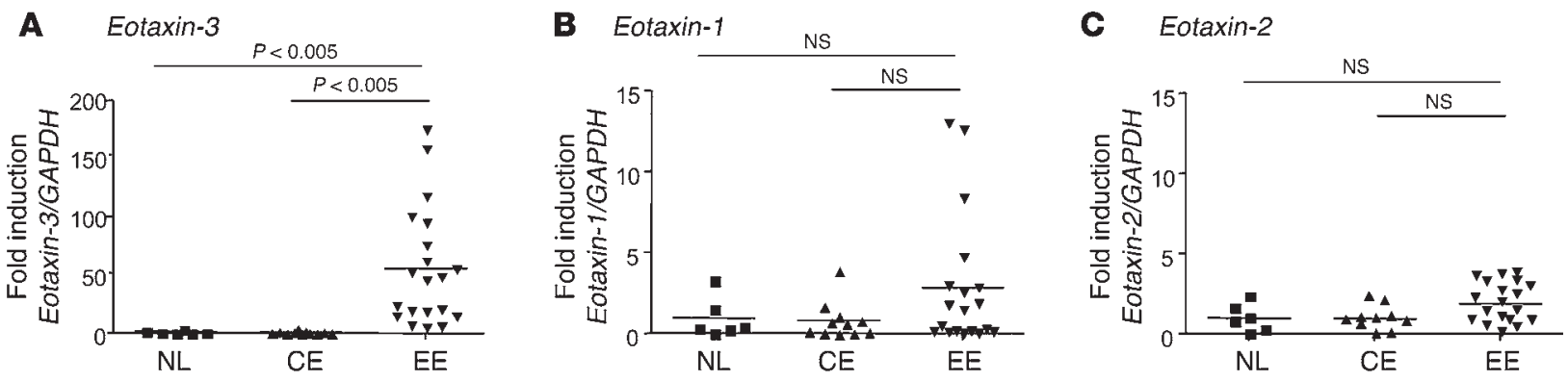

Figure 5

Eotaxin expression in EE patients. Quantitative analysis of eotaxin-1, -2, and -3 mRNA levels in NL, CE, and EE patients using real-time PCR analysis. The level of eotaxin-3 (A), eotaxin-1 (B), and eotaxin-2 (C) mRNA is shown. Each mRNA value is normalized to GAPDH mRNA and is expressed as fold change. The black line represents the mean value in each group. $P$ values were calculated using the Welch $T$ test. The number of subjects was 6,11 , and 19 for NL, CE, and EE, respectively.

In order to localize the eotaxin-3 production in the esophagus, in situ hybridization was performed. As shown in Figure 7, the eotaxin-3 antisense probe yielded a strong signal in the epithelial cell layer of EE patients only. Bright-field microscopy revealed that the eotaxin-3 signal was confined to a population of mononuclear epithelioid cells within the mucosa. The localization of the eotaxin3 was always in epithelioid cells, whose locations were usually close to the proliferative region (basal layer) of the esophagus. Infiltrative eosinophils were eotaxin-3 negative, as were subepithelial structures such as esophageal papillae and underlying stroma. No signal was found in the biopsies of NL patients (Figure 7, A and $\mathrm{B}$ ) or CE patients (data not shown). Hybridization of a control eotaxin-3 sense probe to biopsies of EE, CE, and NL patients revealed no significant background signal (Figure 7, G and H, and data not shown).

Eotaxin-3 protein level in the esophagus and in the blood. Eotaxin-3 protein levels were quantified in NL, CE, and EE patients in esophageal biopsies. Eotaxin-3 protein level in the esophagus of EE patients was significantly increased compared with that in NL and CE patients (Figure 8). In EE patients, the eotaxin-3 protein level was $580 \pm 316 \mathrm{pg} / \mathrm{mg}$ protein (mean $\pm \mathrm{SD}$ ). Indeed, the eotaxin-3 protein level correlated with esophageal level of eosinophils $\left(r^{2}=0.74\right.$, $P<0.05$ ) (data not shown). The esophageal eotaxin-3 protein levels in NL and CE patient biopsies were below the detection limit of the ELISA ( $6 \mathrm{pg} / \mathrm{mg}$ protein). Eotaxin-3 protein level was also quantified in the plasma. A 2-fold increase in eotaxin-3 protein levels was observed between NL and EE plasma samples. Eotaxin-3 protein levels were $64 \pm 36,21 \pm 9$, and $25 \pm 21 \mathrm{pg} / \mathrm{ml}$ in EE, CE, and NL plasma samples, respectively (mean $\pm \mathrm{SD}, P<0.005$ ). Interestingly, while detectable in NL patient biopsies, eotaxin-1 and eotaxin-2 protein levels $(13.6 \pm 11.5$ and $18.4 \pm 12.7 \mathrm{pg} / \mathrm{mg}$ protein, respectively, mean $\pm \mathrm{SD}, n=4$ ) were not significantly increased in CE $(8.1 \pm 16.3$ and $23.3 \pm 18.8 \mathrm{pg} / \mathrm{mg}$ protein, $n=6)$ and EE samples $(33.2 \pm 30.4$ and $27.6 \pm 21.8 \mathrm{pg} / \mathrm{mg}$ protein).

Eotaxin-3 SNP frequency associates with EE. We hypothesized that polymorphism(s) in the eotaxin-3 gene might be associated with disease susceptibility. We checked the position and the frequency of known SNPs in the eotaxin-3 gene (promoter, exons, and untranslated regions) that were between $5 \%$ and $20 \%$ frequency in the white population using the public SNP databanks (http:// www.hapmap.org; and PubMed SNP databanks, http://www.ncbi. nlm.nih.gov). In the promoter, we found 3 SNPs, but they did not modify or create the consensus sequence of a responsive element.
No SNP had a frequency between $5 \%$ and $20 \%$ in the coding region of exons. In the $5^{\prime}$ untranslated region, no SNP matched our criteria. In the $3^{\prime}$ untranslated region, one SNP (rs2302009, 2,496 $T \rightarrow G$ ) was present in $20 \%$ of the white population. Therefore, we genotyped this SNP in patients with EE and control individuals without EE (Table 1). Genotypes of the SNP 2,496 $T \rightarrow G$ were in Hardy-Weinberg equilibrium in both EE patients and unrelated controls. Notably, the allele $G$ was overrepresented in patients with EE compared with race/ethnicity-matched controls (32.1\% versus $22.4 \%, P=0.0069$ ). Additionally, the frequency for genotype $G G$ was significantly higher in patients with $\mathrm{EE}$ (odds ratio 4.55, 95\% confidence interval $1.71-12.39, P=0.001$ ). The $G G$ genotype was not predominant in atopic EE patients; of the 16 GG individuals identified, there were 7 and 9 allergen-sensitized and nonsensi-

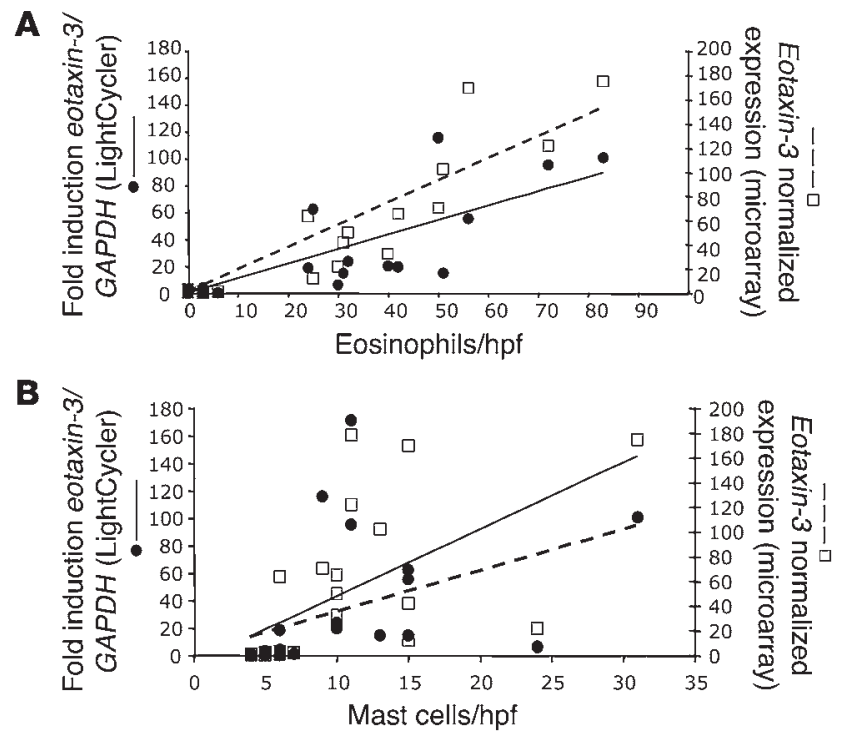

\section{Figure 6}

Correlation between eotaxin-3 mRNA expression and esophageal eosinophil and mast cell counts. The eotaxin-3 expression was measured using LightCycler (filled circles and solid lines, $r^{2}=0.65, P<0.0001$, and $r^{2}=0.18, P<0.05$, in $\mathbf{A}$ and $\mathbf{B}$, respectively) and microarray analysis (open squares and dashed lines, $r^{2}=0.84, P<0.0001$, and $r^{2}=0.36$, $P<0.005$, in $\mathbf{A}$ and $\mathbf{B}$, respectively) and is plotted as a function of the maximum eosinophil (A) and mast cell counts (B) (cells per hpf) present in the biopsies of NL, CE, and EE patients. 


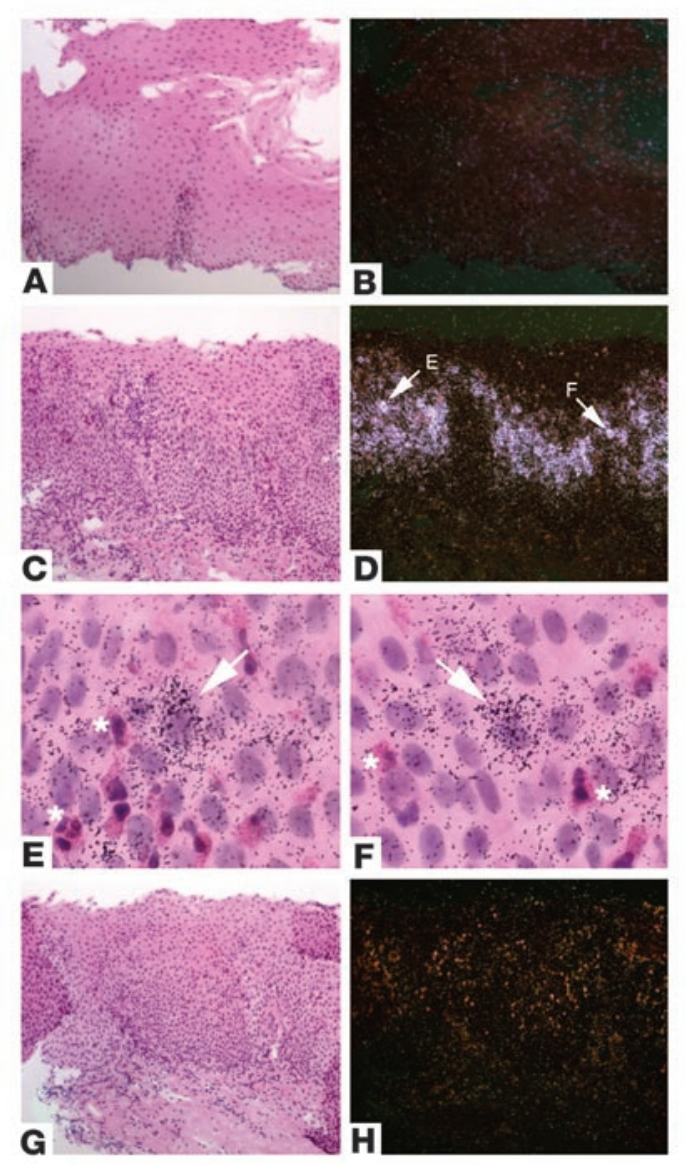

tized individuals, respectively. This suggests that the association of this SNP with EE is not dependent on atopic status. In order to validate the case-control results that could generate false positives due to population stratification, a family-based transmission disequilibrium test was conducted (27). From heterozygous parents, the allele $G$ was preferentially transmitted to affected individuals compared with the alternative allele $T$ (39 versus $18, P=0.0054$ ). The odds ratio was 2.13 (95\% confidence interval 1.57-2.69). Taken together, the results obtained from both case-control and familybased association analyses suggest that the eotaxin-3 gene may be associated with susceptibility to EE.

CCR3 gene-targeted mice are protected from experimental EE. We were interested in testing the importance of the eotaxin pathway directly in vivo. In order to examine this, we turned our attention to the murine system, since an experimental model of EE has been developed. Since an exact homolog of human eotaxin-3 has not yet been characterized in mice, we examined induction of experimental EE in the mice deficient in the eotaxin receptor CCR3. In these experiments, cohorts of wild-type and CCR3-deficient mice were exposed to repeated doses of intranasal allergen under conditions that induce experimental EE. As shown in Figure 9, in wild-type mice, large numbers of eosinophils accumulated in the esophagus. In contrast, CCR3-deficient mice were nearly completely protected from development of esophageal eosinophilia.

\section{Discussion}

$\mathrm{EE}$ is an emerging worldwide disease, yet there is little information concerning its underlying pathogenesis. As such, EE poses

\section{Figure 7}

Eotaxin-3 mRNA expression in biopsies of NL and EE esophagus. Esophageal sections ( $6 \mathrm{NL}, 6 \mathrm{EE}$, and $3 \mathrm{CE}$ ) were subjected to in situ hybridization using an eotaxin-3 antisense probe $(\mathbf{A}-\mathbf{F})$ and sense probe ( $\mathbf{G}$ and $\mathbf{H})$. The hybridization signal of the eotaxin-3 probe is shown in a representative biopsy from an NL patient representative of $6 \mathrm{NL}$ biopsies (A and $\mathbf{B}$ ) and from an EE patient representative of $6 \mathrm{EE}$ biopsies (C-H). Bright-field (A, C, and E-G) and dark-field (B, D, and H) images were photographed at original magnifications of $\times 100$ (A-D, $\mathbf{G}$, and $\mathbf{H}$ ) and $\times 1,000$ (E and $\mathbf{F})$. Arrows indicate eotaxin-3 expression in epithelioid cells (signal grains appear bright in dark-field images and dark in bright-field images), and asterisks indicate representative eosinophils. Red signals in B, D, and $\mathbf{H}$ correspond to autofluorescence, especially of eosinophils.

considerable diagnostic and therapeutic challenges, especially because esophageal eosinophilia has been associated with several other medical conditions, including GERD, parasitic infection, and hypereosinophilic syndromes $(8,28)$. In this study, several principles have emerged. Notably, we have identified a striking EE transcript signature involving approximately $1 \%$ of the human genome. This transcriptome is remarkably conserved between patients despite their age, sex, and allergic status. The apparent homogeneity is even more striking considering that EE has been described as having a non-uniform (i.e., patchy) distribution. Despite the presence of apparent atopic and nonatopic variants of EE, our results indicate that the downstream effector phase of the disease is conserved between these disease variants. This was a surprising finding, because, from the outset, we were concerned that there would be large variability in gene transcript levels among patients because of their divergent clinical presentations (including age and sex). Thus, despite millions of SNPs in the human genome, our results suggest that this complex disorder may have largely conserved disease mechanisms. This finding provides encouraging insight that relatively uniform successful pharmacological therapy may be achieved for EE. Our results are consistent with prior analysis of atopic and nonatopic variants of eosinophilic lung disease (asthma) (29); atopic and nonatopic patients have been shown to have the same cytokine mRNA expression in lung tissue. In our study, based on analysis of hundreds of genes, we present strong evidence that allergic and nonallergic variants of eosinophilic disorders have a common underlying pathogenesis. To our knowledge, this is the first time this type of analysis has been used to examine the etiology of allergic and nonallergic disease variants.

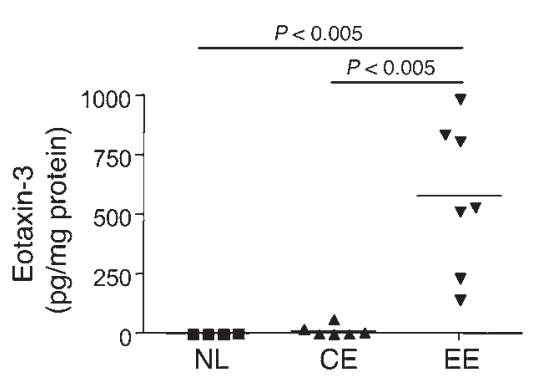

\section{Figure 8}

Eotaxin-3 protein expression. The eotaxin-3 level was assessed by ELISA. Each data point represents the eotaxin-3 level in 1 individual. $P$ values were calculated using the Welch $T$ test. 


\section{Table 1}

Genotype and allele frequencies of the SNP $+2,496 T \rightarrow G$ of the eotaxin-3 gene in EE patients and unrelated individuals without known EE

\begin{tabular}{|c|c|c|c|c|}
\hline SNP +2,496 & $\begin{array}{l}\text { Patients with } \mathrm{EE}^{\mathrm{A}} \\
\qquad(n=117)\end{array}$ & $\begin{array}{c}\text { Unrelated individuals } \\
\text { (without known EE) }(n=225)\end{array}$ & $\begin{array}{c}\text { Odds ratio ( } 95 \% \\
\text { confidence interval) }\end{array}$ & $P$ value \\
\hline \multicolumn{5}{|c|}{ Genotype frequency } \\
\hline$T T$ & $58(49.6 \%)$ & $132(58.7 \%)$ & 1.00 & NS \\
\hline$T G$ & $43(36.8 \%)$ & $85(37.8 \%)$ & $1.15(0.69-1.91)$ & NS \\
\hline$G G$ & $16(13.7 \%)$ & $8(3.6 \%)$ & 4.55 (1.71-12.39) & $0.0010^{\mathrm{B}}$ \\
\hline$T G+G G$ & $59(50.4 \%)$ & $93(41.3 \%)$ & $1.44(0.90-2.32)$ & NS \\
\hline \multicolumn{5}{|c|}{ Allele frequency } \\
\hline$T$ & $159(67.9 \%)$ & $349(77.6 \%)$ & 1.00 & $0.0069^{B}$ \\
\hline G & $75(32.1 \%)$ & $101(22.4 \%)$ & $1.63(1.13-2.35)$ & $0.0069^{B}$ \\
\hline
\end{tabular}

AThere is an overlap of $13 \%$ between the EE patients in this table and the EE patients in Supplemental Table 1. BStatistical significance was determined by exact test using shuffling approach; the $P$ value was generated by $10^{4}$ random permutations of the data.

which eotaxin-3 $(+2,496 T \rightarrow G)$ contributes to EE. Notably, the induction of inflammatory cytokines is often controlled at the level of mRNA stability (32); this appears to be important in glucocorticoid-induced eotaxin-1 downregulation (33), raising the possibility that responsiveness to glucocorticoids in EE could be influenced by eotaxin-3 $(+2,496$ $T \rightarrow G)$. Indeed, a preliminary report has implicated eotaxin-3 mRNA stability in regulating the level of this gene product in epithelial cells (34). At present, of the $19 \mathrm{EE}$ patients who were analyzed for eotaxin-3 production (Supplemental Table

Importantly, our study identified eotaxin-3 as the gene most highly induced in EE. Given the role of this chemokine in regulating CCR3-expressing cell responses in vitro (e.g., eosinophil and mast cell) (30), we focused our attention on this gene product. Indeed, levels of eotaxin-3 expression in the esophagus strongly correlated with disease severity based on basal layer expansion and levels of eosinophils and mast cells. In order to further prove the importance of the identified pathway in vivo, mice with genetic deletion of the eotaxin receptor (CCR3) were shown to be protected from the development of experimental EE. While the mouse data are not directly comparable to the human data because of the different eotaxin genes in mice and humans, this experiment emphasizes the crucial role of CCR3 ligands in experimental EE. This result also indicates that other chemoattractants such as leukotrienes are not likely to have a dominant role in EE. The specific overexpression of eotaxin-3 (and not eotaxin-1 or eotaxin-2) is consistent with prior studies showing the absence of eotaxin-1 overexpression in EE patients (18). The reason that eotaxin-3 is specifically overexpressed compared with eotaxin-1 and eotaxin- 2 deserves further study. It is interesting to note that a recent study has shown eotaxin-3 production by Th2 cytokine-stimulated skin keratinocytes, cells that share properties with esophageal epithelial cells (31). Indeed, other Th2-induced genes were increased in EE (e.g., SOCS, cytokine-inducible SH2 domain-containing protein-1, and IL-8). Although IL-13 has been shown to be overexpressed in EE patients (18), Th2 cytokine mRNA (e.g., IL-4 or IL-13) was not upregulated in EE. Perhaps these cytokine mRNAs might be produced by cells before they infiltrate the tissue or are present in such a low quantity that they are not detectable based on microarray profiling of whole-tissue RNA. Notably, Supplemental Table 3 shows that 54 transcripts are associated with external stress, suggesting that an external stimulus or injury may also induce eotaxin-3. Indeed, eotaxin-3 has been shown to be induced by TNF- $\alpha$ (31). It is thus interesting to speculate that eotaxin-3 may be induced in response to innate signaling, perhaps triggered by ingested stimuli.

We demonstrated that a specific genetic variation in the eotaxin-3 gene is likely associated with EE. This SNP $(+2,496 T \rightarrow G$, rs2302009) locates at the $3^{\prime}$ untranslated region of the eotaxin-3 gene. Modification of mRNA stability may be the mechanism by
1), 9 patients had a $T G$ genotype and none had a GG genotype. Because only the GG genotype was associated with EE in the case-control analysis (Table 1), we would not expect a phenotypic difference between TG and TT; indeed, no phenotypic difference was observed (Supplemental Table 1 and data not shown). This shows that the genetic regulation of eotaxin-3 by this SNP does not universally occur in EE. As in other polygenic complex genetic disorders, such as asthma, the individual contributions of a myriad of genes are likely to be involved in EE (35). This SNP is in complete linkage disequilibrium with another SNP (rs7787623) that is approximately $3 \mathrm{~kb}$ upstream of the eotaxin-3 gene (www. hapmap.org). This indicates that any one of the SNPs tracks with the other and that this polymorphism at the promoter or control region of the eotaxin-3 gene, either alone or in combination with other markers, may be functionally important in EE. Recently, the $S N P+2,496$ has been reported to be associated with atopy in the Korean population $(36,37)$. However, the frequency for allele $G$ in our white normal control population is considerably higher than in normal Koreans (21\% versus 5\%) (www.hapmap.org). This large allele frequency difference across human subpopulations is another indication that this SNP may be functionally important (38). Hopefully, this genetic finding may be used in combination

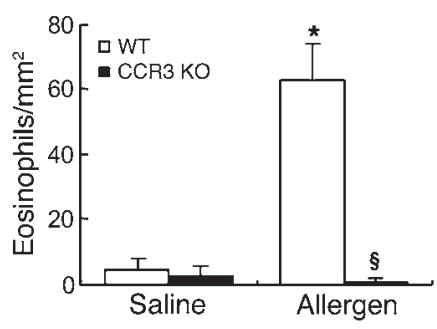

\section{Figure 9}

Role of CCR3 in allergen-induced eosinophil recruitment to the esophagus of wild-type and CCR3-deficient (KO) mice. Mice were challenged with saline or allergen intranasally 3 times a week for 3 weeks. The esophagus was harvested 24 hours after the last intranasal treatment, and esophageal sections were stained with anti-MBP. Results represent the number of eosinophils (mean $\pm S D, n=3$ ) present in the esophagus per square millimeter. ${ }^{\star} P<0.05$ versus saline group, $\S P<0.05$ versus wild-type group. 


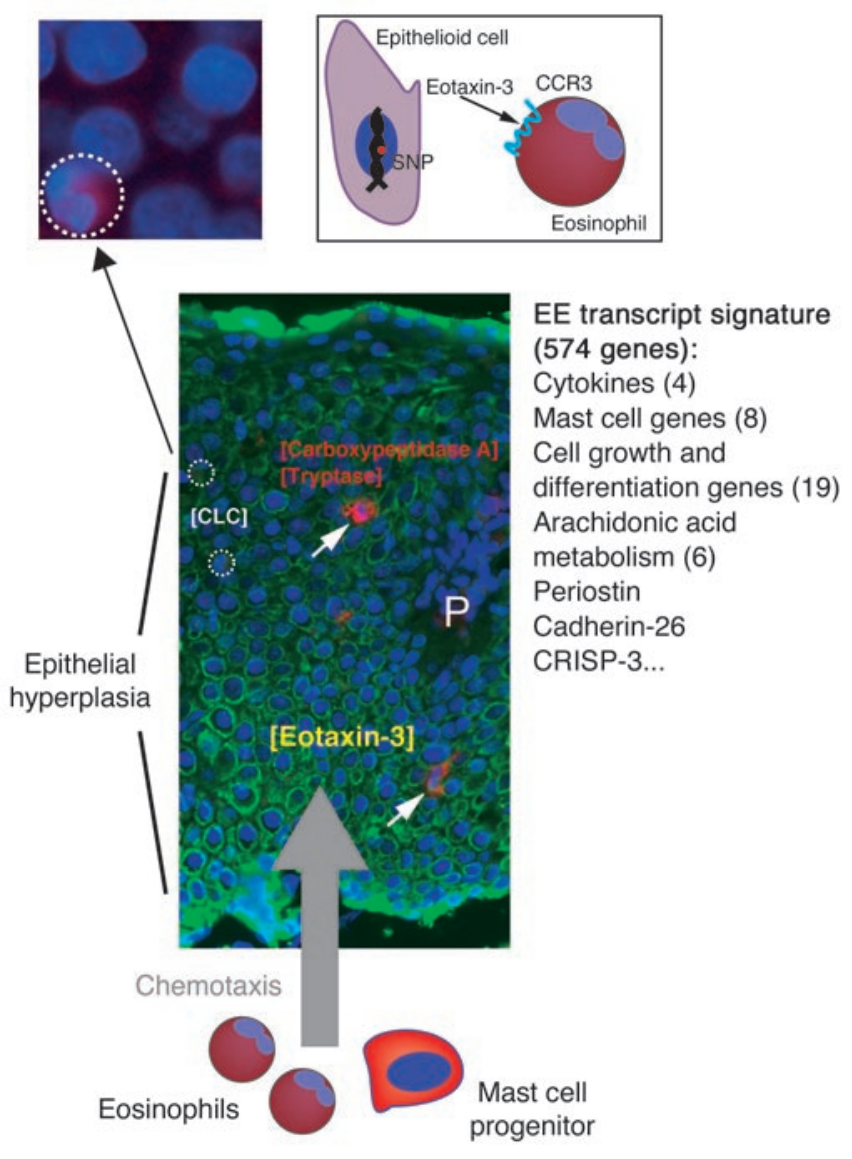

with other markers (including eotaxin-3 protein levels) to establish noninvasive ways of assessing disease risk and/or phenotype.

While not the primary focus of this study, it has not escaped our attention that $\mathrm{CE}$ is also characterized by its own unique transcript signature. Although we did not directly study GERD, the CE pathology was typical of GERD and is thus likely applicable to GERD, at least in part. It will be important to correlate the identified transcript changes with esophageal $\mathrm{pH}$ monitoring. Notably, GERD has not yet been analyzed by DNA microarray analysis. Our study is strongly dependent on the histological diagnosis (e.g., eosinophil level); however, while there was a modest degree of overlap in CE and EE genes, there was a striking difference in the magnitude of the gene changes, the number of genes modified, and, more importantly, the type of dysregulated genes. Taken together, this analysis demonstrates that EE and CE are quite separate diseases and are unlikely to represent a continuum of esophagitis. Further analysis of other CE patients (particularly those with higher eosinophil levels) will be important. Our results provide potential diagnostic criteria for distinguishing EE from other types of esophagitis. Levels of the genes listed in Figure 3 may indeed be critical disease determinants.

Our microarray analysis draws attention to the involvement of mast cells in EE based on the dominant mast cell gene signature. Interestingly, mast cells and mast cell genes were upregulated when eosinophil levels reached 24 eosinophils per hpf, suggesting that mast cell accumulation correlates very strongly with eosinophils. Indeed, mast cells have been previously reported to be elevated in the esophagus of EE patients, although no assessment of their

\section{Figure 10}

Cellular and molecular mediators in EE. Microscopic assessment (lower panel; magnification, $\times 100$ ) using a tryptase-specific antibody demonstrates scattered mast cells (bright-red-fluorescent cells marked by white arrows) among cytokeratin-positive epithelial cells (green-fluorescent cells, which are appropriately absent from the fibrovascular stroma within a papilla, marked "P"). Two eosinophils are designated by dashed circles. Eosinophils are identified by their characteristic red autofluorescence and nuclear morphology under higher magnification (e.g., lower left cell in top left panel; magnification, $\times 1000$; green channel omitted). Nuclei are fluorescently counterstained (blue) with DAPI. We propose a model of EE pathogenesis involving eotaxin-3 expression by epithelioid cells. Eotaxin-3 overexpression promotes chemoattraction of CCR3-positive eosinophils and expression of the CLC protein. An SNP in the eotaxin-3 gene is associated with EE. Mast cells (white arrows) accumulate in the esophagus, and mast cell genes (tryptase- $\alpha$ and carboxypeptidase A3) are overrepresented in the EE transcript signature (Supplemental Table 5). Eotaxin-3 drives eosinophil activation that leads to tissue damage. CRISP-3, cysteinerich secretory protein-3.

genetic content or phenotype has been made (18). Our finding of tryptase expression (Figure 10) without chymase suggests the involvement of T cell-dependent mucosal mast cells (39). It is important to note that the level of mast cells also correlated with disease severity based on eosinophil counts and the degree of epithelial basal layer expansion. It is interesting to note that mast cells also express CCR3 and respond to CCR3 ligands (40-42). Thus, eotaxin-3 may also target mast cells in EE. Our results highlight the need to further characterize the involvement of mast cells in EE and focus attention on the potential benefit of anti-mast cell therapy for the treatment of EE.

Our data indicate a dysregulation of transcripts that primarily reflects the epithelial tissue (likely epithelial cells). Our primary interest was not to identify eosinophil transcripts, but rather diseased tissue transcripts that may explain pathogenesis, at least in part. Our analysis provides a new view on EE, since we propose that the disease involves a problem extrinsic to eosinophils involving overproduction of eotaxin-3 (and other gene products) by resident cells within the epithelium. Few eosinophil-derived gene products were present in the EE transcript signature; major basic protein $(M B P)$ (1.5-fold change), eosinophil-derived neurotoxin (0.85-fold change), eosinophil peroxidase (0.94-fold change), and CCR3 (1.4-fold change) were absent in the signature. This may be due to the dilution of eosinophil transcripts with transcripts from relatively RNA-rich cells such as epithelial cells, fibroblasts, and mast cells. An absence of eosinophil transcript signature has been previously observed in murine models of asthma (43) and also in human atopic dermatitis lesions (44), even though these tissues also have abundant eosinophils. However, the Charcot-Leyden crystal (CLC) mRNA, an eosinophil-specific transcript, was dramatically overexpressed in EE. CLCs, along with other eosinophil products, likely promote proinflammatory changes including epithelial hyperplasia $(16,45-47)$. It is tempting to speculate about the significance of numerous other genes in the EE transcript signature (Figure 10). For example, periostin, a gene that is strongly overexpressed (47-fold) in EE patients, has been associated with epithelial cell growth, angiogenesis, and cellular adhesion $(48,49)$. Also of interest, cadherin-26 (overexpressed 23-fold in EE patients) is a member of the cadherin family of molecules that has been associated with a variety of inflammatory and epithelial 
proliferation diseases. The most downregulated gene, CRISP-3 (cysteine-rich secretory protein-3), is an androgen-dependent transcript (50), perhaps linking the male gender predominance in EE. In EE patient biopsies, there was a profound dysregulation in genes involved in arachidonic acid metabolism (e.g., upregulation of 15-lipoxygenase and downregulation of 12-lipoxygenase). Interestingly, products of arachidonic acid metabolism have been shown to affect Th2 cytokine production or epithelial cell growth $(51,52)$. While a limited number of other chemokines, such as CXCL1, CXCL6, and CXCL8, were induced in EE, these chemoattractants were not associated with their characteristic neutrophil accumulation in EE (data not shown). Perhaps molecules such as TNFAIP6 (53-55), induced 23-fold in EE, block neutrophil infiltration. It is interesting to note that eotaxin-3 is an antagonist of CCR1, CCR2, and CCR5 $(56,57)$, and this may inhibit the action of these chemokine receptors and other cell types. Other CCR3positive cells may include dendritic cells and mast cells $(58,59)$, and these cells have indeed been shown to be increased in our study (Figure 2) and in EE in the literature $(13,18)$. Interestingly, Ig isotypes were found to be dramatically increased (more than 10-fold), emphasizing the potential of in situ B cell development and Ig secretion in the inflamed tissue of EE patients. The EE transcripts do not appear to represent an alteration in cell signature alone. For example, some mast cell genes are increased 2-fold (chymase), whereas others are increased 6-fold (tryptase) or 20-fold (carboxypetidase A3), showing a dissociation from the 3-fold change in mast cell levels. Similarly, CLC protein is increased 20-fold, yet other eosinophil-specific genes are not increased. Epithelial cell hyperplasia may explain some of the increased gene levels; however, these genes are not present in CE patients even though CE also demonstrates epithelial hyperplasia. Despite the prominent epithelial hyperplasia observed in EE, numerous epithelial cellspecific genes (such as esophagin, esophagus cancer-related gene-2, and filaggrin) are decreased, suggesting a modification of the nature of the epithelium. Collectively, these findings support the view that the EE transcript profile reflects transcriptional dysregulation rather than simply cell signature changes. Taken together, our results draw attention to a variety of pathways that deserve further attention for etiopathogenesis and treatment.

These results provide unprecedented insight into the molecular aspects of EE, providing new targets for EE treatment strategies. In particular, we propose that EE is an eotaxin-3-associated disease and involves a markedly conserved genetic transcript signature (Figure 10). The magnitude of gene changes in EE compared with CE supports the notion that EE is a primary esophageal disease. Based on these results, we are hopeful that eotaxin-3 and/or CCR3 blockers may be beneficial for the treatment of EE. These findings are likely to contribute to prediction of the general outcome of EE and to the building of a molecular classification for diagnosis and therapy of esophagitis.

\section{Methods}

Esophageal samples. The patient characteristics are provided in Supplemental Table 1. Our population (patients 1-37) was selected without any regard to age, atopic status, or sex; samples from all the patients are included in Figure 5. Of these 37 patients, 24 were selected for microarray analysis, based on their diverse clinical features. None of the patients was taking glucocorticoids (topical or oral) at the time of the endoscopy or was on diet treatment; glucocorticoids and diet modification were considered exclusion criteria in this study. Patient biopsies, collected from the distal esoph- agus less than $5 \mathrm{~cm}$ from the lower esophageal sphincter, were submerged in formalin for routine pathological analysis with $\mathrm{H} \& \mathrm{E}$ staining. Diagnosis was established based on the maximum eosinophil count per hpf $(\times 400)$, and basal layer expansion was established according to established criteria $(6,9,11)$. Normal (NL) patients were defined as having 0 eosinophils per hpf and no basal layer expansion. The NL biopsies were obtained from patients who presented with symptoms typical of GERD and EE but were found to have completely normal endoscopic appearance and microscopic analysis. Typically, these patients are labeled as having functional abdominal pain. While these patients may not be completely normal, since they had gastrointestinal symptoms, they serve as a relevant control group for comparison with EE. Patients with CE were defined as having mild expansion of the basal layer (less than approximately one-third of epithelium) and/or no more than 23 eosinophils per hpf. EE patients were defined by at least 24 eosinophils per hpf and extensive basal layer hyperplasia (expansion to more than approximately one-third of epithelium). The maximum eosinophil and lymphocyte counts and thickness of the basal layer were assessed after H\&E staining; using well-oriented transverse sections, the thickness of the basal layer was assessed by the number of cells containing a high-density nucleus. This study was approved by the Institutional Review Board of the Cincinnati Children's Hospital Medical Center.

Assessment of allergen sensitization. Skin-prick testing was performed for a panel of 11 aeroallergens and 63 food antigens and assessed based on a $0-4$ scale by comparison with the histamine control response. A score greater than or equal to 2 was considered positive. The number of positive skin-prick tests is provided in Supplemental Table 1. Patients with at least 1 positive skin-prick test were considered to be allergen sensitive. History of past or present atopic dermatitis, allergic rhinitis, eczema, or asthma is shown in Supplemental Table 1. The Pharmacia Immuno CAP System (Pharmacia Diagnostics) was used to quantify levels of food allergen-specific IgE levels (referred to as RAST, or RadioAllergoSorbent test); values greater than $0.35 \mathrm{kU} / 1$ were considered positive.

DNA microarray analysis. For each patient, 1 distal esophageal mucosal biopsy sample was immersed in RNAlater RNA stabilization reagent (QIAGEN) and stored at $4^{\circ} \mathrm{C}$ for less than 15 days. Total RNA was extracted using RNeasy Mini Kit (QIAGEN) according to the manufacturer's recommendations. Hybridization to DNA microarray was performed by the Microarray Core at Cincinnati Children's Hospital Medical Center, as previously reported (43). The genome-wide human Affymetrix U133 Plus 2.0 GeneChip was used, and gene transcript levels were determined using algorithms in the Microarray Analysis Suite and GeneSpring software (Silicon Genetics).

Ontology assessment. We subjected the list of differentially expressed transcripts to gene ontology analysis using DAVID (Database for Annotation, Visualization and Integrated Discovery) and EASE (Expression Analysis Systematic Explorer), Web-based applications (http://david. niaid.nih.gov/david/upload.asp) that allow access to a relational database of functional annotations $(60,61)$.

In situ hybridization. Esophageal biopsy samples were fixed in $4 \%$ paraformaldehyde/PBS, stored overnight at $4{ }^{\circ} \mathrm{C}$, and subsequently submerged in $30 \%$ sucrose (43). In brief, eotaxin- 3 cDNA was generated using the primers ACCTGAGAAGGGCCTGATTT and GTAACTCTGGGAGGAAACACCCTCTCC and cloned into PCR2.2 vector (Invitrogen Corp.). The resulting plasmid was linearized by BamHI or XhoI digestion, and sense and antisense RNA probes, respectively, were generated by $\mathrm{T} 7$ and sp6 RNA polymerase (Riboprobe System Kit; Promega). The radiolabeled $(\alpha 35$ SthioUTP) probes were hybridized, slides were washed under highstringency conditions, and autoradiography was performed for 2-4 weeks at $4^{\circ} \mathrm{C}$. The specificity of the hybridization was established using the eotaxin-3 sense riboprobe. Sections from NL, EE, and CE patients were hybridized and underwent autoradiography under identical conditions. 
Real-time PCR analysis. The RNA samples (500 ng) were subjected to reverse transcription analysis using Iscript reverse transcriptase (Bio-Rad Laboratories) according to manufacturer's instructions. Eotaxin-1, eotaxin-2, and eotaxin-3 were quantified by real-time PCR using the LightCycler instrument and LightCycler FastStart DNA Master SYBR Green I as a ready-to-use reaction mix (Roche Diagnostics Corp.). Results were then normalized to GAPDH amplified from the same cDNA mix and expressed as fold induction compared with the controls. cDNAs were amplified using the following primers: buman eotaxin-3 (151 bp), AACTCCGAAACAATTGTACTCAGCTG and GTAACTCTGGGAGGAAACACCCTCTCC; buman eotaxin-2 (251 bp), CCATAGTAACCAGCCTTC and CAGGTTCTTCATGTACCTC; human eotaxin-1 (425 bp), TGAAGCTTGGGCCAGCTTCTGTCCCAACC and GGTCGACTGGAGTTGGAGATTTTTGGTC; GAPDH (400 bp), TGGAAATCCCATCACCATCT and GTCTTCTGGGTGGCAGTGAT.

Eotaxin protein determinations. Plasma from heparinized blood was extracted, and the eotaxin-3 protein level in $100 \mu$ l of plasma was quantified using Quantikine kit CCL26 (R\&D Systems) according to the manufacturer's instructions. Results are expressed as picograms of eotaxin-3 per milliliter plasma \pm SD. For esophageal eotaxin protein levels, methods previously reported were used (62). Briefly, esophageal biopsies were immersed in RNAlater RNA stabilization reagent, washed twice in PBS, and homogenized in $200 \mu \mathrm{l}$ acetic acid $(2 \mathrm{M})$ /hydrochloric acid ( $0.1 \mathrm{M})$. Samples were boiled for 10 minutes and neutralized to $\mathrm{pH} 7$ with $35 \mu \mathrm{l}$ of ammonium hydroxide (30\% wt/vol) (62). Eotaxin-1, -2, and -3 levels were quantified using DuoSet kits (R\&D Systems). The detection limits for eotaxin-1, eotaxin-2, and eotaxin-3 were each $7 \mathrm{pg} / \mathrm{ml}$. The recovery of eotaxin-3, spiked into NL esophageal tissue before the extraction procedure, was $76 \%$. The total protein level was assayed in the samples using BCA Protein Assay Reagent (Pierce). Results are expressed as picograms eotaxin-3 per milligram protein (mean $\pm \mathrm{SD}$ ).

SNP analysis. Buccal swab DNA was collected from EE patients $(n=117)$, their parents $(n=134)$, and an unrelated set of white individuals without known EE $(n=225)$ after informed consent. Briefly, our EE population was $72.3 \%$ male, $76.2 \%$ allergen sensitized, and $100 \%$ white. Moreover, the age range of this population was 3 months to 23 years with a mean of 8.5 years, and eosinophils were observed in the proximal esophagus in $84.4 \%$ of these patients; thus our population is representative of the EE population previously described $(2,5,9,18,45)$. DNA was isolated by alkaline extraction. SNP detection was accessed using a LightCycler instrument (Roche Diagnostics Corp.). PCR was performed with LightCycler FastStart DNA Master Hybridization Probes (Roche Diagnostics Corp.) using the eotaxin-3specific primers AAGGAAAAAATGGGTGCA and TGAACAACCTTTATTAAAGTAACTCT. For eotaxin-3 SNP analysis, the anchor probe was labeled with LCred640 linked to AGCCAAGAGCGGGGTCC. The sensor probe (GCGTCCTCGGATGACAATTCA) was labeled with fluorescein and designed to span the $\mathrm{T} \rightarrow \mathrm{G}$ mutation.

Immunohistochemistry and immunofluorescence. Esophageal sections were immunostained with anti-tryptase antibody. Briefly, endogenous peroxidases were quenched with $0.3 \%$ hydrogen peroxide in methanol followed by a treatment with Trilogy with EDTA (Cell Marque) for 17 minutes in a steamer. Tissue sections were then incubated with the prediluted monoclonal mouse anti-tryptase antibody CMA890 (Cell Marque) for 1 hour at room temperature. Then, the LSAB+/HRP kit K0679 and DAB Enhancer (Dako) were used according to the manufacturer's protocol. Slides were then counterstained with hematoxylin (Zymed Laboratories Inc.) for 5 minutes. Immunoreactive cells were counted $(\times 400)$ and are expressed as maximum mast cell number per hpf.

For immunofluorescence microscopy, slide-mounted cryosections were air dried and acetone fixed, washed in PBS, incubated in a blocking solution containing $2 \%$ goat serum, and then incubated $\left(18\right.$ hours at $\left.4{ }^{\circ} \mathrm{C}\right)$ with diluted, biotinylated anti-mast cell tryptase (Promega) primary antibody.
Sections were then washed with PBS and incubated (1:200, 30 minutes at $25^{\circ} \mathrm{C}$ ) with Alexa Fluor 594-labeled streptavidin (Invitrogen Corp.). After washing with PBS, slides were incubated $\left(45\right.$ minutes at $25^{\circ} \mathrm{C}$ ) with diluted anti-cytokeratin (Dako) primary antibody. Sections were then washed with PBS and incubated $\left(1: 200,30\right.$ minutes at $\left.25^{\circ} \mathrm{C}\right)$ with Alexa Fluor 488-labeled goat anti-rabbit secondary antibody (Invitrogen Corp.). After washing with PBS, slides were coverslipped using antifade medium containing DAPI (ProLong Gold; Invitrogen Corp.) and photographed using an RT Slider digital camera (Diagnostic Instruments).

Experimental allergen-induced EE in mice. $\mathrm{BALB} / \mathrm{c}$ mice (National Cancer Institute) and CCR3-deficient mice (BALB/c background; a kind gift of A. Humbles and C. Gerard, Harvard Medical School, Boston, Massachusetts, USA) were housed under specific pathogen-free conditions. Experimental EE was induced by exposure of mice to Aspergillus fumigatus antigen intranasally 3 times a week for 3 weeks as previously described (16). Mice were sacrificed 48 hours after the last challenge, and the esophagus was harvested and fixed in formalin. Eosinophil levels were determined by immunostaining for mouse eosinophil major basic protein (anti-MBP; a kind gift of J. Lee, Mayo Clinic, Scottsdale, Arizona, USA), as previously reported (16).

Statistics. Gene lists on microarray were obtained by study of differences in gene-expression levels between groups using the Welch $T$ Test and 2-tailed Student's $t$ test (with or without Benjamini and Hochberg false discovery rate [FDR] correction). The EE transcript was obtained using the Welch $T$ test with FDR $(P \leq 0.01)$. The CE transcript signature was composed of the addition of the gene lists from the Welch $T$ test without FDR and genes from the Student's $t$ test without FDR $(P \leq 0.01)$. Genes differently expressed between allergic and nonallergic EE were composed of the addition of the gene lists from the Welch and Student's $t$ tests without FDR $(P \leq 0.05)$. Ordered tree clustering was performed using standard correlation or distance. Correlation of gene expression with numeric clinical parameters or eosinophil levels was assessed using the Pearson correlation test with $P$ value. Tests used to generate the gene lists and the number of genes in these lists are shown in Supplemental Table 2. These lists were filtered based on $P$ value and/or fold changes. Statistical significance between groups of data was determined using an unpaired 2-tail Welch $T$ test or Mann-Whitney $U$ test with Bonferroni correction, and correlations of data with the number of eosinophils in the biopsies were determined using the Pearson correlation test with $P$ value. $P$ values less than or equal to 0.05 were considered statistically significant.

A case-control comparison was conducted at both genotype and allele frequency levels ( $\mathrm{rs} 2302009$, SNP 2,496T $\rightarrow G$ ), where the cases were from the proband of each family and a set of race/ethnicity-matched unrelated healthy individuals was collected as controls. The statistical significance was evaluated by exact test using a shuffling method, generated by $10^{4}$ random permutations of the data. The Hardy-Weinberg equilibrium test, which compares the observed genotype with the expected genotype, was also conducted in cases and controls, respectively, using the $\chi^{2}$ test (63). Next, the association between the SNP 2,497T $\rightarrow G$ and EE susceptibility was examined by the family-based transmission disequilibrium test to determine whether the affected child received the disease-associated allele more frequently than the alternative allele. The software TDT/S-TDT, version 1.1 (64), was used for analysis (65).

\section{Acknowledgments}

The authors wish to thank Andrea Lippelman for assistance with the preparation of this manuscript, Christine Fischetti, Chris Woods, and Betsy Dipasquale for their technical assistance, and Huan Xu for her assistance with the microarray analysis. We also thank James Heubi, Michelle Lierl, Kathleen Cambell, and Ranajit Chakraborty for their helpful insight and/or referral of patients. 
We are grateful to Alison Humbles and Craig Gerard for supplying CCR3-deficient mice, and Jamie Lee for anti-MBP reagent. This work was supported in part by the Burroughs Wellcome Fund and the Food Allergy \& Anaphylaxis Network (to M.E. Rothenberg), NIH grants R01 AI45898 (to M.E. Rothenberg) and R24 DK064403 (to M.B. Cohen), and the CURED (Campaign Urging Research for Eosinophilic Disease) Foundation.
Received for publication August 24, 2005, and accepted in revised form November 29, 2005.

Address correspondence to: Marc Rothenberg, Division of Allergy and Immunology, Cincinnati Children's Hospital Medical Center, 3333 Burnet Avenue, Cincinnati, Ohio 45229, USA. Phone: (513) 636-7210; Fax: (513) 636-3310; E-mail: Rothenberg@cchmc.org.
1. Fox, V.L., Nurko, S., and Furuta, G.T. 2002. Eosinophilic esophagitis: it's not just kid's stuff. Gastroin test. Endosc. 56:260-270.

2. Straumann, A., et al. 2003. Natural history of primary eosinophilic esophagitis: a follow-up of 30 adult patients for up to 11.5 years. Gastroenterology. 125:1660-1669.

3. Orenstein, S.R., et al. 2000. The spectrum of pediatric eosinophilic esophagitis beyond infancy: a clinical series of 30 children. Am. J. Gastroenterol. 95:1422-1430

4. Cheung, K.M., Oliver, M.R., Cameron, D.J., CattoSmith, A.G., and Chow, C.W. 2003. Esophageal eosinophilia in children with dysphagia. J. Pediatr. Gastroenterol. Nutr. 37:498-503.

5. Noel, R.J., Putnam, P.E., and Rothenberg, M.E. 2004. Eosinophilic esophagitis. N. Engl. J. Med. 351:940-941.

6. Kelly, K.J., et al. 1995. Eosinophilic esophagitis attributed to gastroesophageal reflux: improvement with an amino acid-based formula. Gastroenterology. 109:1503-1512.

7. Walsh, S.V., et al. 1999. Allergic esophagitis in children: a clinicopathological entity. Am. J. Surg. Pathol. 23:390-396.

8. Rothenberg, M.E. 2004. Eosinophilic gastrointestinal disorders (EGID) [review]. J. Allergy Clin. Immunol. 113:11-28.

9. Sant'Anna, A.M., Rolland, S., Fournet, J.C., Yazbeck, S., and Drouin, E. 2004. Eosinophilic esophagitis in children: symptoms, histology and $\mathrm{pH}$ probe results. J. Pediatr. Gastroenterol. Nutr. 39:373-377.

10. Lim, J.R., et al. 2004. White specks in the esophageal mucosa: an endoscopic manifestation of nonreflux eosinophilic esophagitis in children. Gastrointest. Endosc. 59:835-838.

11. Ruchelli, E., Wenner, W., Voytek, T., Brown, K., and Liacouras, C. 1999. Severity of esophageal eosinophilia predicts response to conventional gastroesophageal reflux therapy. Pediatr. Dev. Pathol. 2:15-18.

12. Attwood, S.E., et al. 2003. Eosinophilic oesophagitis: a novel treatment using Montelukast. Gut. 52:181-185.

13. Teitelbaum, J.E., et al. 2002. Eosinophilic esophagitis in children: immunopathological analysis and response to fluticasone propionate. Gastroenterology. 122:1216-1225.

14. Mishra, A., Hogan, S.P., Lee, J.J., Foster, P.S., and Rothenberg, M.E. 1999. Fundamental signals that regulate eosinophil homing to the gastrointestinal tract. J. Clin. Invest. 103:1719-1727.

15. Rothenberg, M.E., Mishra, A., Brandt, E.B., and Hogan, S.P. 2001. Gastrointestinal eosinophils. Immunol. Rev. 179:139-155.

16. Mishra, A., Hogan, S.P., Brandt, E.B., and Rothenberg, M.E. 2001. An etiological role for aeroallergens and eosinophils in experimental esophagitis. J. Clin. Invest. 107:83-90.

17. Mishra, A., Hogan, S.P., Brandt, E.B., and Rothenberg, M.E. 2002. IL-5 promotes eosinophil trafficking to the esophagus. J. Immunol. 168:2464-2469.

18. Straumann, A., Bauer, M., Fischer, B., Blaser, K., and Simon, H.U. 2001. Idiopathic eosinophilic esophagitis is associated with a $\mathrm{T}(\mathrm{H}) 2$-type allergic inflammatory response. J. Allergy Clin. Immunol. 108:954-961.

19. Mishra, A., and Rothenberg, M.E. 2003. Intratra- cheal IL-13 induces eosinophilic esophagitis by an IL-5, eotaxin-1, and STAT6-dependent mechanism. Gastroenterology. 125:1419-1427.

20. Garrett, J.K., et al. 2004. Anti-interleukin-5 (mepolizumab) therapy for hypereosinophilic syndromes. J. Allergy Clin. Immunol. 113:115-119.

21. Schmid-Grendelmeier, P., et al. 2002. Eosinophils express functional IL-13 in eosinophilic inflammatory diseases. J. Immunol. 169:1021-1027.

22. Komiya, A., et al. 2003. Concerted expression of eotaxin-1, eotaxin-2, and eotaxin-3 in human bronchial epithelial cells. Cell. Immunol. 225:91-100.

23. Ying, S., et al. 1999. Eosinophil chemotactic chemokines (eotaxin, eotaxin-2, RANTES, monocyte chemoattractant protein-3 (MCP-3), and MCP-4), and $\mathrm{C}-\mathrm{C}$ chemokine receptor 3 expression in bronchial biopsies from atopic and nonatopic (Intrinsic) asthmatics. J. Immunol. 163:6321-6329.

24. Rankin, S.M., Conroy, D.M., and Williams, T.J. 2000. Eotaxin and eosinophil recruitment: implications for human disease. Mol. Med. Today. 6:20-27.

25. Gerard, C., and Rollins, B.J. 2001. Chemokines and disease. Nat. Immunol. 2:108-115.

26. Al-Tubuly, A.A., Spijker, R., Pignatelli, M., Kirkland, S.C., and Ritter, M.A. 1997. Inhibition of growth and enhancement of differentiation of colorectal carcinoma cell lines by MAb MR6 and IL-4. Int. J. Cancer. 71:605-611.

27. Gordon, D., and Finch, S.J. 2005. Factors affecting statistical power in the detection of genetic association. J. Clin. Invest. 115:1408-1418. doi:10.1172/ JCI24756.

28. Shah, U., and Walker, W.A. 2002. Pathophysiology of intestinal food allergy. Adv. Pediatr. 49:299-316.

29. Humbert, M., et al. 1996. IL-4 and IL-5 mRNA and protein in bronchial biopsies from patients with atopic and nonatopic asthma: evidence against "intrinsic" asthma being a distinct immunopathologic entity. Am. J. Respir. Crit. Care Med. 154:1497-1504.

30. Kitaura, M., et al. 1999. Molecular cloning of a novel human CC chemokine (Eotaxin-3) that is a functional ligand of CC chemokine receptor 3 . J. Biol. Chem. 274:27975-27980.

31. Kagami, S., et al. 2005. Interleukin-4 and interleukin-13 enhance CCL26 production in a human keratinocyte cell line, HaCaT cells. Clin. Exp. Immu nol. 141:459-466.

32. Atasoy, U., et al. 2003. Regulation of eotaxin gene expression by TNF-alpha and IL-4 through mRNA stabilization: involvement of the RNA-binding protein HuR. J. Immunol. 171:4369-4378.

33. Stellato, C., et al. 1999. Differential regulation of epithelial-derived C-C chemokine expression by IL-4 and the glucocorticoid budesonide. J. Immunol. 163:5624-5632.

34. Heller, N.M., et al. 2004. Interferon-gamma inhibits STAT6 signal transduction and gene expression in human airway epithelial cells. Am. J. Respir. Cell Mol. Biol. 31:573-582.

35. Hoffjan, S., Nicolae, D., and Ober, C. 2003. Association studies for asthma and atopic diseases: a comprehensive review of the literature. Respir. Res. 4:14.

36. Chae, S.C., Park, Y.R., Oh, G.J., Lee, J.H., and Chung, H.T. 2005. The suggestive association of eotaxin2 and eotaxin- 3 gene polymorphisms in Korean population with allergic rhinitis. Immunogenetics. 56:760-764.

37. Chae, S.C., et al. 2004. Analysis of the polymor- phisms in eotaxin gene family and their association with asthma, IgE, and eosinophil. Biochem. Biophys. Res. Commun. 320:131-137.

38. Akey, J.M., Zhang, G., Zhang, K., Jin, L., and Shriver, M.D. 2002. Interrogating a high-density SNP map for signatures of natural selection. Genome Res. 12:1805-1814.

39. Austen, K.F., and Boyce, J.A. 2001. Mast cell lineage development and phenotypic regulation. Leuk. Res. 25:511-518.

40. Romagnani, P., et al. 1999. Tryptase-chymase double-positive human mast cells express the eotaxin receptor CCR 3 and are attracted by CCR3-binding chemokines. Am. J. Pathol. 155:1195-1204.

41. de Paulis, A., et al. 2001. Expression of the chemokine receptor CCR3 on human mast cells. Int. Arch. Allergy Immunol. 124:146-150.

42. Ochi, H., et al. 1999. T helper cell type 2 cytokinemediated comitogenic responses and CCR 3 expression during differentiation of human mast cells in vitro. J. Exp. Med. 190:267-280.

43. Zimmermann, N., et al. 2003. Dissection of experimental asthma with DNA microarray analysis identifies arginase in asthma pathogenesis. J. Clin. Invest. 111:1863-1874. doi:10.1172/JCI24756.

44. Nomura, I., et al. 2003. Distinct patterns of gene expression in the skin lesions of atopic dermatitis and psoriasis: a gene microarray analysis. J. Allergy Clin. Immunol. 112:1195-1202.

45. Noel, R.J., et al. 2004. Clinical and immunopathologic effects of swallowed fluticasone for eosinophilic esophagitis. Clin. Gastroenterol. Hepatol. 2:568-575.

46. Gleich, G.J. 2000. Mechanisms of eosinophilassociated inflammation. J. Allergy Clin. Immunol. 105:651-663.

47. Weller, P.F. 1997. Human eosinophils. J. Allergy Clin. Immunol. 100:283-287.

48. Tai, I.T., Dai, M., and Chen, L.B. 2005. Periostin induction in tumor cell line explants and inhibition of in-vitro cell growth by anti-periostin antibodies. Carcinogenesis. 26:908-915.

49. Shao, R., and Guo, X. 2004. Human microvascular endothelial cells immortalized with human telomerase catalytic protein: a model for the study of in vitro angiogenesis. Biochem. Biophys. Res. Commun. 321:788-794.

50. Haendler, B., Kratzschmar, J., Theuring, F., and Schleuning, W.D. 1993. Transcripts for cysteinerich secretory protein-1 (CRISP-1; DE/AEG) and the novel related CRISP- 3 are expressed under androgen control in the mouse salivary gland. Endocrinology. 133:192-198.

51. Carey, M.A., et al. 2003. Accentuated T helper type 2 airway response after allergen challenge in cyclooxygenase-1-/- but not cyclooxygenase-2-/mice. Am. J. Respir. Crit. Care Med. 167:1509-1515.

52. Sheng, G.G., et al. 1997. A selective cyclooxygenase 2 inhibitor suppresses the growth of H-ras-transformed rat intestinal epithelial cells. Gastroenterology. 113:1883-1891.

53. Cao, T.V., La, M., Getting, S.J., Day, A.J., and Perretti, M. 2004. Inhibitory effects of TSG-6 Link module on leukocyte-endothelial cell interactions in vitro and in vivo. Microcirculation. 11:615-624.

54. Szanto, S., Bardos, T., Gal, I., Glant, T.T., and Mikecz, K. 2004. Enhanced neutrophil extravasation and rapid progression of proteoglycan-induced 
arthritis in TSG-6-knockout mice. Arthritis Rheum. 50:3012-3022.

55. Getting, S.J., et al. 2002. The link module from human TSG-6 inhibits neutrophil migration in a hyaluronan- and inter-alpha-inhibitor-independent manner. J. Biol. Chem. 277:51068-51076.

56. Ogilvie, P., Paoletti, S., Clark-Lewis, I., and Uguccioni, M. 2003. Eotaxin-3 is a natural antagonist for CCR2 and exerts a repulsive effect on human monocytes. Blood. 102:789-794.

57. Petkovic, V., Moghini, C., Paoletti, S., Uguccioni, M., and Gerber, B. 2004. Eotaxin-3/CCL26 is a natural antagonist for $\mathrm{CC}$ chemokine receptors 1 and 5. A human chemokine with a regulatory role. J. Biol. Chem. 279:23357-23363.
58. Humbles, A.A., et al. 2002. The murine CCR3 receptor regulates both the role of eosinophils and mast cells in allergen-induced airway inflammation and hyperresponsiveness. Proc. Natl. Acad. Sci. U. S. A 99:1479-1484.

59. Beaulieu, S., et al. 2002. Expression of a functional eotaxin (CC chemokine ligand 11) receptor CCR3 by human dendritic cells. J. Immunol. 169:2925-2936.

60. Dennis, G., Jr., et al. 2003. DAVID: Database for Annotation, Visualization, and Integrated Discovery. Genome Biol. 4:P3

61. Hosack, D.A., Dennis, G., Jr., Sherman, B.T., Lane, H.C., and Lempicki, R.A. 2003. Identifying biological themes within lists of genes with EASE. Genome
Biol. 4:R70.

62. Blanchard, C., et al. 2005. Eotaxin-3/CCL26 gene expression in intestinal epithelial cells is up-regulated by interleukin- 4 and interleukin- 13 via the signal transducer and activator of transcription 6 . Int. J. Biochem. Cell Biol. 37:2559-2573.

63. Ott, J. 2005. Linkage utility programs for human genetics. http://linkage.rockefeller.edu/ott/ linkutil.htm.

64. TDT/S-TDT. http://genomics.med.upenn.edu/ spielman/TDT.htm.

65. Spielman, R.S., and Ewens, W.J. 1998. A sibship test for linkage in the presence of association: the sib transmission/disequilibrium test. Am. J. Hum. Genet. 62:450-458. 